\title{
miR-17 inhibits ovarian cancer cell peritoneal metastasis by targeting ITGA5 and ITGB1
}

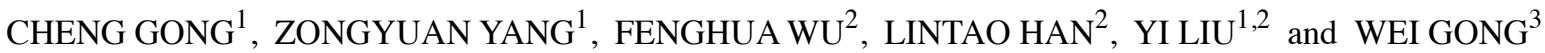 \\ ${ }^{1}$ Department of Obsterics and Gynecology, Tongji Hospital, Tongji Medical College, Huazhong University of Science and \\ Technology, Wuhan, Hubei 430010; ${ }^{2}$ Department of Medicinal Chemistry, School of Pharmacy, Hubei University of \\ Chinese Medicine, Wuhan, Hubei 430065; ${ }^{3}$ Department of Oncology, Xiang Yang Central Hospital, \\ Hubei University of Arts and Science, XiangYang, Hubei 441021, P.R. China
}

Received February 6, 2016; Accepted June 21, 2016

DOI: $10.3892 /$ or.2016.4985

\begin{abstract}
An essential step in the peritoneal spread of ovarian cancer is the adhesion and implantation of tumor cells to the mesothelium layer. Integrin $\alpha 5$ and $\beta 1$ have been reported to mediate the initial adhesion process and to correlate with disease survival in ovarian cancer. However, the molecular mechanism of integrin $\alpha 5 \beta 1$ dysregulation in tumorigenesis and metastasis remained enigmatic. In the present study, using the US NCI60 database, we identified miR-17 as a candidate regulator targeting both integrin $\alpha 5$ and $\beta 1$. The level of miR-17 was evidently inversely correlated with that of $\alpha 5$ and $\beta 1$ in ovarian cancer cell lines. Specifically, miR-17 bound directly to the $3^{\prime}$ untranslated region (3'UTR) of $\alpha 5$ and $\beta 1$ and suppressed their expression. Forced expression of miR-17 led to markedly diminished adhesion and invasion of ovarian cancer cells in vitro, and notably reduced metastatic nodules inside the peritoneal cavity in in vivo SKOV3 xenografts model. Moreover, ectopic expression of miR-17 in ovarian cancer cells resulted in repressed ILK phosphorylation as well as decreased production of active matrix metalloproteinase-2 (MMP-2). Our results indicated that miR-17 hampered ovarian cancer peritoneal propagation by targeting integrin $\alpha 5$ and $\beta 1$. These findings supported the utility of miR-17/ $\alpha 5 \beta 1$ to be considered as valuable marker for metastatic potential of ovarian cancer cells, or a therapeutic target in ovarian cancer treatment.
\end{abstract}

Correspondence to: Professor Wei Gong, Department of Oncology, XiangYang Central Hospital, Hubei University of Arts and Science, XiangYang, Hubei 441021, P.R. China

E-mail: gongzhewei@sina.com

Professor Yi Liu, Department of Medicinal Chemistry, School of Pharmacy, Hubei University of Chinese Medicine, Wuhan, Hubei 430065, P.R. China

E-mail:23218843@qq.com

Key words: miR-17, integrin $\alpha 5 \beta 1$, adhesion, peritoneal metastasis, ovarian cancer

\section{Introduction}

Majority of patients with epithelial ovarian carcinoma (EOC) are not diagnosed until advanced stage (III or IV) characterized by widespread peritoneal dissemination and ascites. Along with rapid occurrence of chemoresistance, this brings down the 5-year survival of EOC to $30 \%$ (1). Ovarian tumor cells primarily exfoliate from primary site and shed into the peritoneal cavity where they can implant onto the mesothelial layer covering the abdominal organ, which is a crucial step in the formation of secondary foci and EOC progression (2). Identifying critical molecules involved in the interaction between the exfoliated tumor cells and the peritoneal microenvironment may not only advance our understanding of the disease, but may ultimately enable us to develop effective therapeutic interventions.

It is generally accepted that the initial step of ovarian cancer metastasis is adhesion of cancer cells to the mesothelium lining the peritoneum, mesentery and omentum (3). Impeding this attachment process could be a way of blocking ovarian cancer dissemination. The mechanistic explanation concerning the adherence progress remains poorly elucidated. Integrins that mediated binding to extracellular matrix (ECM) elements such as fibronectin (Fn), laminin and collagen, and CD44 that facilitated binding to hyaluronan expressed on the surface of mesothelium were identified as key orchestrators (4,5). Among the integrin family, integrin $\alpha 5$ (ITGA5) and integrin $\beta 1$ (ITGB1) are of particular interest in ovarian cancer malignant behavior (6-8).

Integrin $\alpha 5$ primarily combines with integrin $\beta 1$ subunit to form $\alpha 5 \beta 1$ heterodimer and recognizes its specific ligand Fn, which is one of the most plentiful proteins in ECM of the peritoneal cavity $(9,10)$. Once ovarian cancer cells have detached from the primary site, they float in the ascites as single cells or as multicellular spheroids $(11,12)$. Previous studies revealed that loss of E-cadherin in ovarian cancer cells was accompanied with the elevation of integrin $\alpha 5$, which facilitates colonization of tumor cells at secondary sites (8). Recent research showed that the upregulation of c-Met during tumor progression, contributed to ovarian cancer peritoneal propagation through an integrin $\alpha 5 \beta 1$-dependent mechanism (13). These accumulative findings strongly imply that integrin $\alpha 5 \beta 1$ 
could be a potential therapeutic target, at least for a subset of EOC patients.

A growing body of evidence suggests that miRNAs, small non-coding RNAs 22 nucleotides in length, are capable of regulating gene expression through matching to target genes, either completely or partially, at the 3'-untranslated region (3'UTR), causing the suppression of protein translation or mRNA degradation (14). More than 1,500 different miRNAs have been identified related to cancer development in human, and these miRNAs have the potential to regulate up to $30 \%$ of human genes (15). In the present study, we set to identify the miRNAs that regulate integrin $\alpha 5 \beta 1$ expression thus indirectly dominating ovarian cancer dissemination, and to evaluate the therapeutic potential of targeting this miRNA. Eventually, we identified hsa-mir-17 (miR-17) as a candidate. Overexpression of miR-17 reduced integrin $\alpha 5 \beta 1$ expression in ovarian cancer cells, accompanied with the inhibition of cell adhesion and invasion. In in vivo ovarian cancer xenografts, lentiviral transfection of miR-17 into SKOV3-Luc ovarian cancer cells evidently reduced peritoneal dissemination. These results suggested that miR-17 may be exploited as intervention target for patients with ovarian cancer. Further research may be needed to develop future clinical applications.

\section{Materials and methods}

Cells culture and transfection. The human ovarian cancer cell lines SKOV3, CaOV3, OV-90, OVCAR3, CaOV4, ES2, TOV-21G and Tov-112D were purchased from the American Type Culture Collection (ATCC; Manassas, VA, USA). These cells were maintained in McCoy's 5A, RPMI-1640 medium or Dulbecco's modified Eagle's medium (DMEM) containing $10 \%$ fetal bovine serum (FBS) according to the manual on ATCC website. SKOV3 cells were transduced with CMV-Fluc-IRES-GFP lentiviral plasmid (GeneChem, Shanghai, China) and designated as SKOV3-Luc. GFP-positive cells were enriched through FACS and further used in vivo in adhesion assay and living imaging. Transient transfections of the miR-17 mimics, miR-inhibitor and scrambled negative control (RiboBio, Guangzhou, China) were conducted using Lipofectamine 2000 (Invitrogen, Carlsbad, CA, USA) according to the manufacturer's instructions.

SKOV3-Luc cells were transduced with hsa-mir-17 or mir-control lentivirus particles (GeneChem) according to the manufacturer's protocol, the stable transfected cells were selected by continuous exposure to puromycin (Invitrogen). The transduced cells were designated as SKOV3-Luc-miR-17 and SKOV3-Luc-NC, respectively.

Western blot assay. Western blotting was performed as described in our previous study (16). The Abs of ITGA5, ITGB1, p-ILK, matrix metalloproteinase-2 (MMP-2) and GAPDH were obtained from Epitomics (Burlingame, CA, USA).

RNA extraction and $q R T-P C R$. Total RNAs were extracted from cultured cells using the RNA simple total RNA extraction kit (Tiangen, Beijing, China) according to the manufacturer's instructions. The expression of ITGA5 and ITGB1 was determined by the SYBR-Green qPCR, and GAPDH was used as the loading control. The relative gene expression normalized to GAPDH was quantified using the comparative $\mathrm{C}_{\mathrm{T}}$ method, and expressed as fold-change relative to the control.

miRNA and mRNA correlation of NCI60 database. Normalized miRNA and mRNA data collections of 60 cancer cell lines were obtained from NCI60 online source (http://discover. nci.nih.gov/cellminer/). Pearson's correlation analysis was performed between the expressional levels of hsa-mir-17 and the ITGA5 and ITGB1 probe set, respectively.

Dual luciferase reporter assay. The specific target sequence of miR-17 in the 3'UTR region of human ITGA5 and ITGB1 was predicted with TargetScan (www.TargetScan.org). ITGA5 and ITGB1 3'UTR and their variant sequence with three nucleotides mutated in the miR-17 target site were synthesized and cloned into psi-CHECK2 vector downstream of Rellina to generate the recombinant vectors, psi-CHECK2-ITGA5/ITGB1-3'UTR-wt and psi-CHECK2-ITGA5/ITGB1-3'UTR-mt, respectively. SKOV3 cells were co-transfected with the generated plasmids and miR-17 mimics or miR-17 inhibitor. Luciferase activity was determined using the Dual-Luciferase Reporter Assay System (Promega, Madison, WI, USA) after $48 \mathrm{~h}$ of incubation. Rellina luciferase values were normalized to firefly luciferase values.

Transwell invasion assay. The invasion assay was performed using an $8-\mu \mathrm{m}$ pore size chamber (Corning, Corning, NY, USA) coated with $50 \mu 1$ Matrigel (BD Biosciences, Franklin Lakes, NJ, USA) 1:6 diluted with serum-free DMEM. The lower compartment was filled with $600 \mu \mathrm{l}$ medium containing $20 \%$ FBS as the chemoattractant. A total of $2 \times 10^{4}$ cells $/ 100 \mu$ l were seeded into the upper compartment and incubated for $24 \mathrm{~h}$ at $37^{\circ} \mathrm{C}$. Following removal of the non-migratory cells with a cotton swab, the remaining invaded cells at the lower surface of the filter were fixed with cold methanol for $15 \mathrm{~min}$ and stained with crystal violet for $10 \mathrm{~min}$. The number of invasive cells were calculated by counting 6 random fields at a magnification of x100 (Leica, Solms, Germany).

In vitro and in vivo adhesion assays. For the in vitro adhesion assay, 96-well plates were coated with $10 \mu \mathrm{g} / \mathrm{ml} \mathrm{Fn,} 10 \mu \mathrm{g} / \mathrm{ml}$ type IV collagen or $5 \mu \mathrm{g} / \mathrm{ml}$ laminin in phosphate-buffered saline (PBS) overnight at $4^{\circ} \mathrm{C}$. The wells were thoroughly washed with PBS, and then blocked with $3 \%$ BSA for $1 \mathrm{~h}$ at $37^{\circ} \mathrm{C}$. Approximately $100 \mu \mathrm{l}$ of serum-free medium containing $10^{4}$ cells in each group were added to the coated wells and incubated for up to $1 / 2 \mathrm{~h}$ at $37^{\circ} \mathrm{C}$. The wells were gently rinsed with PBS to remove non-adherent cells. The attached cells were counted at different fields under a light microscope. To study early adhesion of ovarian cancer cells in mouse abdomen, in vivo adhesion assay was performed, $10^{6}$ SKOV3-GFP cells were injected into the peritoneal cavity of female athymic nude mice. Four hours later, mice were sacrificed, small bowel mesenterium and omentum were excised. After the tissues were gently washed with PBS to eliminate non-adherent cells, the adherent cells were observed and photographed under the fluorescence.

Immunohistochemistry. Immunohistochemical staining for ITGA5, ITGB1, p-ILK and MMP-2 expression were 

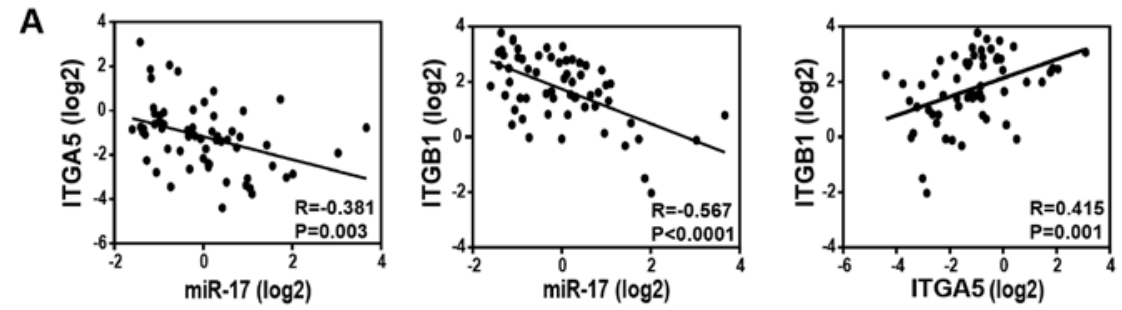

B

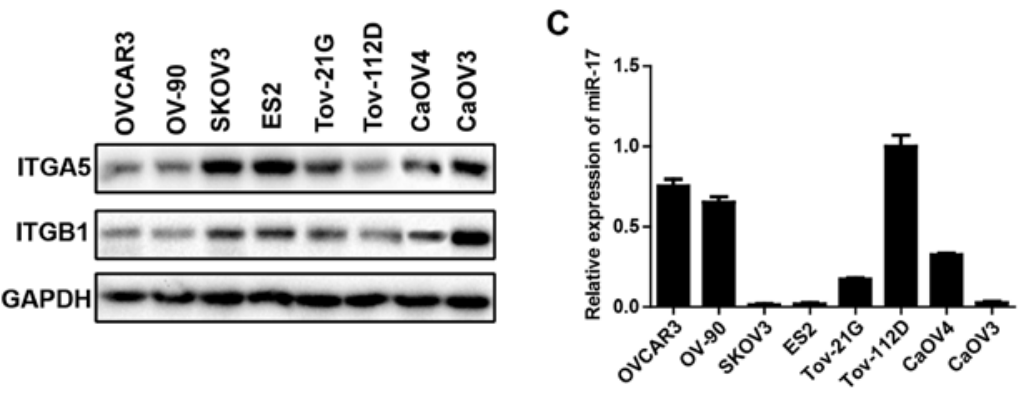

Figure 1. Negative correlation between miR-17 and ITGA5, ITGB1 in ovarian cancer cell lines. (A) Data extracted from NCI60 microarray revealing that the endogenous miR-17 level is inversely correlated with ITGA5 ( $\mathrm{R}=-0.381 ; \mathrm{P}=0.003)$ and ITGB1 $(\mathrm{R}=-0.567 ; \mathrm{P}<0.0001)$ mRNA levels. (B) The expression of ITGA5 and ITGB1 was detected by western blotting in a panel of ovarian cancer cell lines. (C) qRT-PCR detection of miR-17 expression related to U6 in ovarian cancer cell lines.

conducted following standard procedures. Briefly, sections were deparaffinized and then immersed in $3 \% \mathrm{H}_{2} \mathrm{O}_{2}$ to inactivate endogenous peroxidase. The slides were blocked with goat serum for $30 \mathrm{~min}$ and further incubated with the antibody (ITGA5, 1:150; ITGB1, 1:100; p-ILK, 1:50; and MMP-2, 1:50) overnight at $4^{\circ} \mathrm{C}$ after antigen retrieval process. The next day, the slides were exposed to horseradish peroxidase-linked secondary antibody for $20 \mathrm{~min}$. Finally the slides were developed in DAB solution for optimal staining intensity.

Experimental metastasis model. The animal studies were approved by the Committee on the Ethics of Animal Experiments of Tongji Medical College. Female NOD/SCID mice (4-6 weeks old) were purchased from the Beijing HFK Bio-Technology (Beijing, China) and maintained in a laminar flow cabinet under specific pathogen-free conditions. SKOV3-Luc-miR-17 or SKOV3-Luc-NC cells $\left(2 \times 10^{6}\right)$ were injected into peritoneal cavity (10 mice/group). The tumor growth was monitored every 5 days until 30 days from tumor implantation. Mice were anaesthetized with $1 \%$ pentobarbital sodium, and imaged with the IVIS Spectrum System (Caliper; Xenogen, USA) 15 min after intraperitoneal dosage of $100 \mathrm{mg} / \mathrm{kg}$ D-luciferin substrate. Total flux (photons/s) and metastasis localization was analyzed using Living Image version 4.3.1 software.

Statistical analysis. SPSS (version 16.0) software package (SPPS, Inc., Chicago, IL, USA) was used for all the statistical procedures. All the experimental data are presented as means \pm SD. Correlations were analyzed using the Pearson's test. The Student's t-test (two-tailed) was used to determine statistical differences between two experimental groups; difference at $\mathrm{P}<0.05$ was considered to indicate statistical significance.

\section{Results}

Expression level of miR-17 inversely correlates with that of the ITGA5 and ITGB1 in ovarian cancer cell lines. To determine whether the expression of miR-17 and ITGA5 and ITGB1 were correlated, the US NCI60 (National Cancer Institute) database was used, which covers 60 various human cancer cell lines, including five ovarian cancer lines. Data were extracted to analyze the correlation between miR-17 and ITGA5, ITGB1 expression in different types of cancer cell lines. The miR-17 expression level was significantly inversely correlated with ITGA5 ( $\mathrm{R}=-0.381 ; \mathrm{P}=0.003)$ as well as ITGB1 $(\mathrm{R}=-0.567$; $\mathrm{P}<0.0001)$ expression, ITGA5 and ITGB1 are positively correlated $(\mathrm{R}=0.415 ; \mathrm{P}=0.001)$ (Fig. $1 \mathrm{~A})$, strongly suggesting that miR-17 is one of the pivotal regulators of ITGA5 and ITGB1 in cancer cells. Next, we measured ITGA5 and ITGB1 expression by western blotting (Fig. 1B) and miR-17 expression by miRNA RT-PCR (Fig. 1C) in eight different ovarian cancer cell lines. Five (SKOV3, ES2, TOV-21G, CaOV3 and CaOV3) of eight cell lines expressed high levels of ITGA5 and ITGB1, the remaining three (OVCAR3, OV-90 and TOV-112D) expressed relatively low level of ITGA5 and ITGB1. Conversely, miR-17 relative expression levels were found to be significantly lower for the five ovarian cancer cell lines that expressed high levels of ITGA5 and ITGB1. These observations indicated that miR-17 was a likely regulator of ITGA5 and ITGB1 in ovarian cancer cell lines.

ITGA5 and ITGB1 are directly targeted by miR-17 in ovarian cancer cell lines. Based on the above findings, we next set to clarify whether ITGA5 and ITGB1 are direct targets of miR-17. Western blot and qRT-PCR analyses revealed that miR-17 overexpression caused a significant decrease in ITGA5 and ITGB1 mRNA and protein level in SKOV-3 and CaOV3 cells while silencing of miR-17 augments ITGA5 and ITGB1 expression in these cell lines (Fig. 2A and B). To further explore whether miR-17 directly targets the 3'UTR of ITGA5 and ITGB1 mRNA, we performed a dual luciferase reporter assay. The 3'UTR of both ITGA5 and ITGB1 mRNA contains conserved predicted binding site for miR-17, two reporters, one containing wild-type ITGA5 or ITGB1 3'UTR 

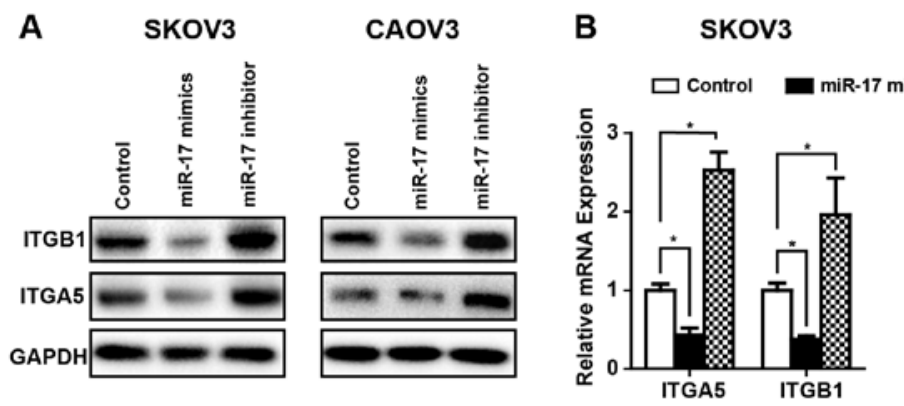

CAOV3

C
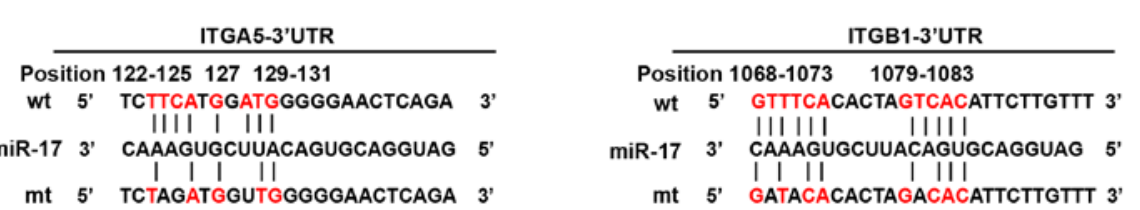

D

SKOV3

CAOV3

$\square \mathrm{NC}=$ miR-17 mimics $\square$ miR-17 inhibitor

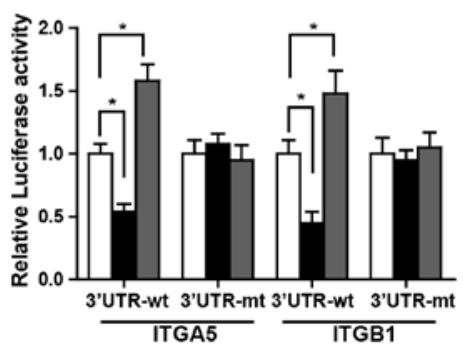

口NC $\square$ miR-17 mimics $\square$ miR-17 inhibitor

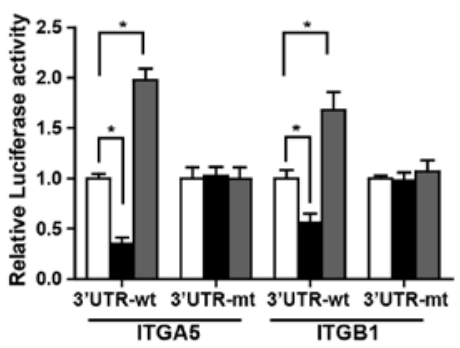

Figure 2. miR-17 directly regulates ITGA5 and ITGB1 expression in ovarian cancer cell lines. SKOV3 and CaOV3 cells were transfected with miR-17 mimics or miR-17 inhibitor for $48 \mathrm{~h}$. (A) Western blot analysis of ITGA5 and ITGB1 with GAPDH as an internal control. (B) Analysis of ITGA5 and ITGB1 mRNA in SKOV3 and CaOV3 cells using qRT-PCR with GAPDH as an internal control. (C) Schematic drawing of miR-17 seed sequence in ITGA5 and ITGB1 3'UTR and constructed mutated 3'UTR. (D) Normalized luciferase activity to Renilla was performed after co-transfection with miR-17 mimics or miR-17 inhibitor and luciferase reporter plasmid containing either wt $3^{\prime} \mathrm{UTR}$ or $\mathrm{mt} 3^{\prime} \mathrm{UTR}$; ${ }^{*} \mathrm{P}<0.05$.

A
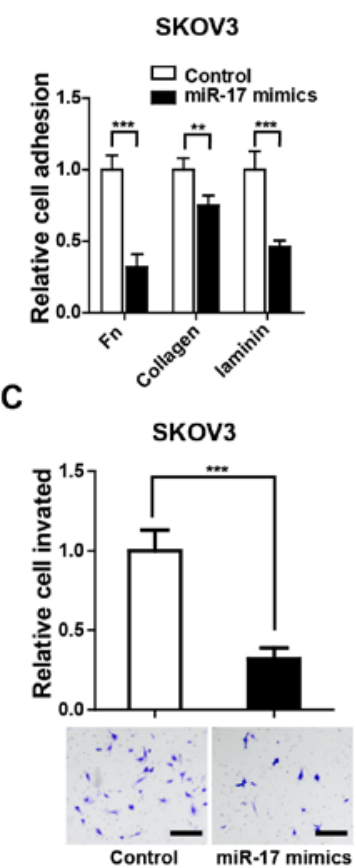

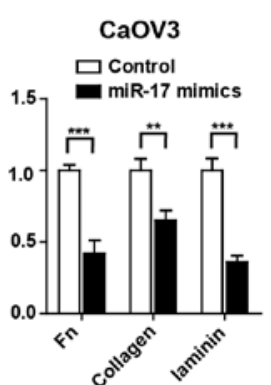

CaOv3

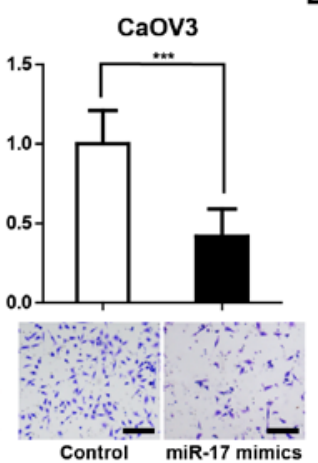

B

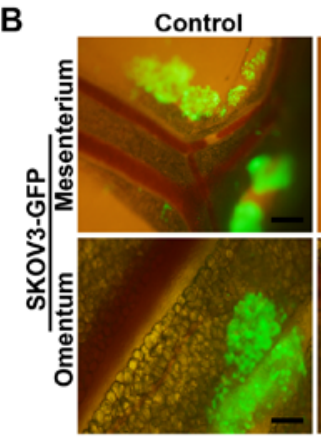

D

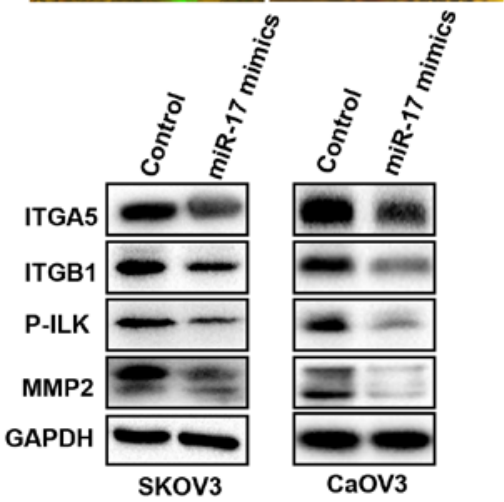

Figure 3. miR-17 overexpression causes remarkable suppression of adhesion and invasion in ovarian cancer cell lines. SKOV3 and CaOV3 cells were transfected with miR-17 mimics for $48 \mathrm{~h}$ before used in following experiments. (A) In vitro adhesion assay determines relative cell number adhere to plates coated with various ECM protein as Fn, collagen and laminin of SKOV3 and CaOV3 cells. (B) SKOV3-GFP cells were allowed to adhere for $4 \mathrm{~h}$ after i.p. injection, representative images of cell aggregates in mesenterium and omentum are shown at a magnification of x100. Bar, $50 \mu \mathrm{m}$. (C) Matrigel invasion assay compares the invasive capacity alteration after miR-17 upregulation, typical graphs are presented at a magnification of x100. Bar, 50 $\mu \mathrm{m}$. (D) Immunoblotting of ITGA5, ITGB1, p-ILK, MMP-2 after $48 \mathrm{~h}$ transfection of miR-17 mimics. GAPDH was used as a loading control; ${ }^{* *} \mathrm{P}<0.01,{ }^{* * *} \mathrm{P}<0.001$. 
A
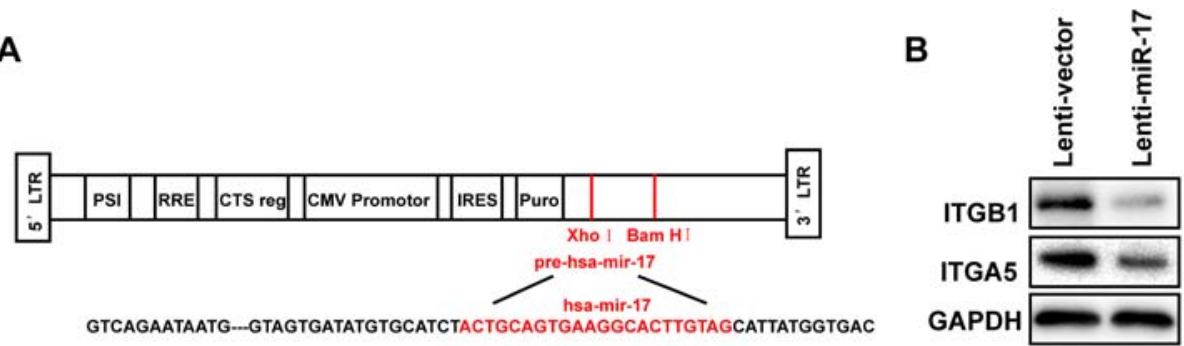

GTCAGAATAATG -..-GTAGTGATATGTGCATCTACTGCAGTGAAGGCACTTGTAGCATTATGGTGAC

GAPDH

C

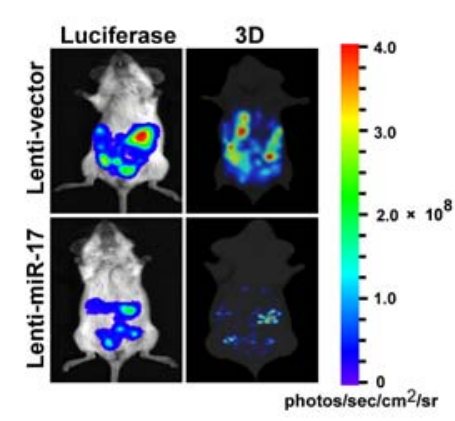

D

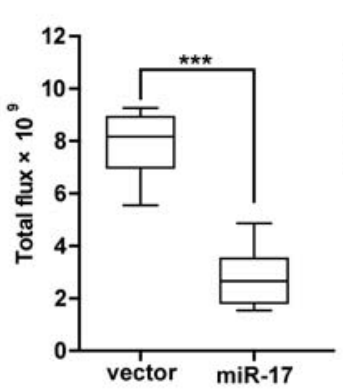

E

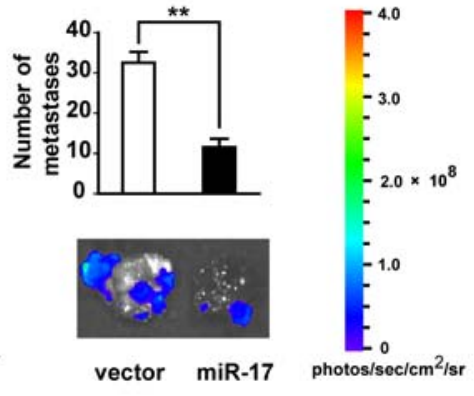

$\mathbf{F}$
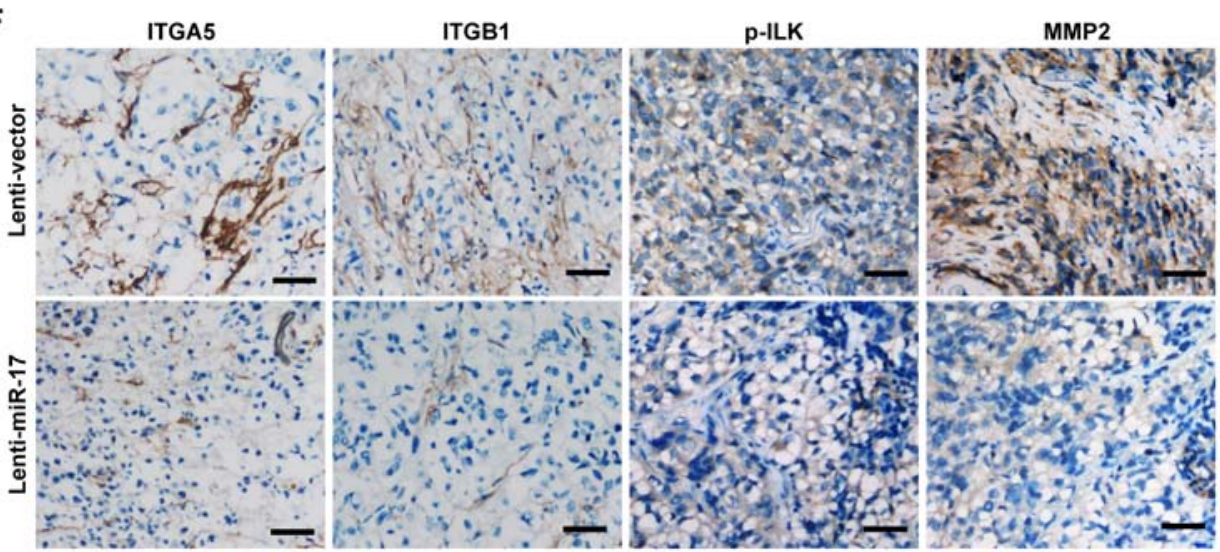

Figure 4. miR-17 hampers peritoneal dissemination of ovarian cancer xenograft model. (A) Illustration of a lentivirus construct expressing pre-miR-17 and a puromycin-resistance gene (rPuro). (B) Western blot analysis of ITGA5 and ITGB1 expression in stably transfected SKOV3 cells. (C and D) After one month inoculation of indicated cells, the development of metastatic lesions in the peritoneal cavity of mice was monitored by in vivo bioluminescence images. Representative image of tumor burden in each group is shown (C). The total luciferase flux was calculated (n=10/group) (D). (E) The average number of metastasis nodules was counted in both group. (F) Immunohistochemical staining of ITGA5, ITGB1, p-ILK and MMP-2 in tumor sections isolated from each group. Bar, $50 \mu \mathrm{m} ;{ }^{* *} \mathrm{P}<0.01,{ }^{* * *} \mathrm{P}<0.001$.

with the miR-17 binding site and another containing mutant 3'UTR were constructed (Fig. 2C). A significant decrease in relative luciferase activity was observed in ovarian cancer cells (SKOV3 and CaOV3) transfected with miR-17 mimics when compared with NC miRNA. In contrast, inhibition of miR-17 caused a notable increase in relative luciferase activity. Nevertheless, both the miR-17 mimics and inhibitor failed to affect the activity of mutant reporter construct, indicating a specific direct interaction of miR-17 with the 3'UTR of ITGA5 and ITGB1 mRNA. Collectively, these data showed a directly suppression of ITGA5 and ITGB1 by miR-17 in ovarian cancer cell lines.

miR-17 suppresses in vitro and in vivo adhesion, invasion capability of ovarian cancer cell lines. Due to the recognized role of ITGA5 and ITGB1 in ovarian cancer progression $(4,8)$, we enforced miR-17 expression in ovarian cancer cells and evaluated the effects on cell adhesion and invasion. miR-17 transfection markedly diminished cell adhesion onto Fn, collagen and laminin in SKOV3 and CaOV3 cells (Fig. 3A). Next, we transferred the adhesion assay into the mice peritoneal cavity, similar finding was observed that miR-17 overexpressed SKOV3-GFP cells presented evident diminished cell aggregates adhesion in mice mesentery and omentum region (Fig. 3B). Since invasion is the subsequent event following adhesion in ovarian cancer metastasis (17). Then, we detected whether miR-17 overexpression also affects the invasive capacity of ovarian cancer cells. Enforced expression of miR-17 significantly impaired the invasion of SKOV3 and CaOV3 cells (Fig. 3C). Remodeling of cell-ECM interactions via integrins provokes a cascade of phosphorylation of signal transduction molecules at focal adhesions (18). Phosphorylation of ILK is known to be particularly crucial in integrin-mediated outside-in signal transduction pathways, 
which regulate various gene expression or cell behavior, such as cell dispersal and migration (19,20). Enforced expression of miR-17 drastically inhibited ITGA5 and ITGB1 expression, and the phosphorylation of ILK, followed by the downregulation of MMP-2, which is believed to play a pivotal role in ovarian cancer invasion and metastasis (Fig. 3D) (21).

miR-17 inhibits peritoneal dissemination in an ovarian cancer xenograft model. In light of the evident inhibitory effect of adhesion and invasion by miR-17 in ovarian cancer cells through repressing ITGA5 and ITGB1 expression, we further looked into the restorative effect of enforced expression of miR-17 in ovarian cancer i.p. implantation xenograft model. Lentiviruses with the miR-17 precursor (Fig. 4A) or scrambled miRNA were constructed and stably transfected into SKOV3-Luc cells. Downregulation of ITGA5 and ITGB1 expression were confirmed after miR-17 overexpression (Fig. 4B).

Tumor cell propagation in the peritoneal cavity of mice were assessed 1 month after i.p. injection, in vivo bioluminescence images showed that mice inoculated with cells that overexpressed miR-17 presented markedly reduced metastatic lesions in the abdomen, particularly on the peritoneal surface, small-bowel mesentery, omentum region with 3D-reconstruction (Fig. 4C). As a result, the total tumor burden in the SKOV3-LUC-miR-17 group was attenuated markedly compared with the SKOV3-LUC-NC group (Fig. 4D). Consistently, the SKOV3-LUC-miR-17 cells also developed more and larger nodules than those generated by SKOV3-LUC-NC cells (Fig. 4E). Immunohistochemical staining analysis also further demonstrated that miR-17 overexpression endowed cells with reduced ITGA5 and ITGB1, as well as weaker p-ILK and MMP-2 expression. Taken together, these in vivo results demonstrated the inhibitory action of miR-17 on the intraperitoneal dissemination of ovarian cancer cells.

\section{Discussion}

Several years of intensive research has elucidated the role of integrin family as a multifunctional ingredient involved in a myriad of tumor cell processes, including adhesion, invasion, ECM remodeling, angiogenesis and metastasis in human cancer (22). In ovarian cancer, integrin $\alpha 5 \beta 1, \alpha v \beta 3$ and $\alpha 2 \beta 1$ are the main integrins that contribute to poor prognosis $(4,9)$. However, integrin $\alpha 5 \beta 1$ was proven to participate in the tumor cell adherence and clearance of mesothelial layer on the peritoneal surfaces (23), which is a crucial step in the establishment of secondary foci, making it a very attractive therapeutic target in ovarian cancer. Moreover, increased integrin $\alpha 5 \beta 1$ levels correlated with chemotherapy resistance and poor patient survival $(3,24)$, while the dysregulation mechanism of integrin $\alpha 5 \beta 1$ in tumorigenesis and metastasis are poorly elucidated to date. Thus, it would be of great interest to explore the possible mechanism of $\alpha 5 \beta 1$ disorder such as miRNAs.

Recent studies have emphasized the pivotal roles of miRNAs in cancer development and progression, they contribute to almost all aspects of cancer biology such as proliferation, apoptosis, angiogenesis and metastasis $(14,15)$. In the present study, using the US database of NCI60, we identified that miR-17 expression was inversely correlated with both integrin $\alpha 5$ and $\beta 1$ levels in human cancer cells. Our experiments further demonstrated the negative correlation between miR-17 and integrin $\alpha 5 \beta 1$ in several ovarian cancer cell lines. This hypothesis was substantially confirmed by our findings that miR-17 directly binds to the 3'UTR region of integrin $\alpha 5$ and $\beta 1$ in a luciferase reporter system. miR-17 belongs to the miR-17-92 cluster, which comprises six members: miR-17, -1 8a, -19a, -20a, -19b-1 and -92a. Previous studies designated the miR-17-92 cluster as cancer driver for their general overexpression in several types of cancer (25). As far as ovarian cancer, it seemed contrasting that a miR-17-92 cluster member miR-92a had been proved to inhibit ovarian cancer peritoneal dissemination (3). However, miR-17 had been shown to promote the proliferation of gastric and cervical cancer cells, and metastasis of osteosarcoma and colorectal cancer (26-29). According to our results, miR-17 suppressed the in vitro and in vivo adhesion of ovarian cancer cells, accompanied by a diminished invasive capacity. These observations made us explore a more comprehensive understanding of the role of miR-17 in ovarian cancer peritoneal metastasis.

Evidence exists of integrin communication with ECM molecules accelerating the adhesion and invasion of cancer cells, and regulating the metastatic potential of tumor cells through integrin-linked kinase (ILK)-mediated signaling (19). The data presented in the present study clearly showed that miR-17 overexpression reduced the protein level of integrin $\alpha 5$ and $\beta 1$, hampering the phosphorylation of ILK, inhibiting production of active MMP-2. Therefore, the miR-17-mediated downregulation of $\alpha 5 \beta 1$ expression not only suppressed the adhesion and invasion of tumor cells, but also blocked ECM- $\alpha 5 \beta 1$-mediated signal transduction to regulate various gene expression in tumor cells. The production of MMPs plays the leading role in integrin-induced remodeling and degradation of ECM, which is indispensible for tumor cell metastasis. Reducing the amount of active MMPs in the tumor niche may be one of the reasons contributing to inhibited $\alpha 5 \beta 1$-mediated invasion of tumor cells exerted by miR-17. These results support the fact that integrin $\alpha 5 \beta 1$ expression was closely associated with ovarian carcinoma invasion involving MMP-2, which was in accordance with several previous studies (30).

It was noteworthy that an ovarian cancer xenograft model was chosen for further confirmation of forced expression of miR-17 influence on peritoneal dissemination. Our present data showed that miR-17 overexpressed SKOV3-Luc cells produced remarkably fewer metastatic lesions, thus reduced the tumor burden inside the abdominal cavity. Consistently, the IHC analysis validated that ectopic expression of miR-17 caused the suppression of integrin $\alpha 5 \beta 1$ as well as downstream p-ILK and active MMP-2 in the tumor sections. In that sense, the inhibitors against integrin $\alpha 5 \beta 1$ apparently indicate the possibility to be developed for clinical use. Treatment of mice with the integrin $\alpha 5 \beta 1$ antibody was reported to inhibit adhesion and peritoneal metastasis of ovarian cancer cells (8). Monoclonal antibody directly against integrin $\alpha 5 \beta 1$ (volociximab) was recently invented and tested in preclinical trials with patients with recurrent ovarian cancer (31). Therefore, miR-17 may emerge as an alternative or more powerful target for controlling ovarian cancer exacerbation.

In summary, our findings emphasized the unanticipated regulation of integrin $\alpha 5$ and integrin $\beta 1$ by miR-17 in 
ovarian cancer cells. Having provided novel evidence that miR-17 inhibited the adhesion and invasion of ovarian cancer cells by repressing $\alpha 5 \beta 1$, the present study suggested that targeting miR-17 generated new possibilities in ovarian cancer treatment through the inhibition of integrin $\alpha 5 \beta 1$.

\section{References}

1. Landen CN Jr, Birrer MJ and Sood AK: Early events in the pathogenesis of epithelial ovarian cancer. J Clin Oncol 26: 995-1005, 2008

2. Engel J, Eckel R, Schubert-Fritschle G, Kerr J, Kuhn W, Diebold J, Kimmig R, Rehbock J and Hölzel D: Moderate progress for ovarian cancer in the last 20 years: Prolongation of survival, but no improvement in the cure rate. Eur J Cancer 38: 2435-2445, 2002.

3. Ohyagi-Hara C, Sawada K, Kamiura S, Tomita Y, Isobe A, Hashimoto K, Kinose Y, Mabuchi S, Hisamatsu T, Takahashi T, et al: miR-92a inhibits peritoneal dissemination of ovarian cancer cells by inhibiting integrin $\alpha 5$ expression. Am J Pathol 182: 1876-1889, 2013.

4. Lessan K, Aguiar DJ, Oegema T, Siebenson L and Skubitz AP: CD44 and beta1 integrin mediate ovarian carcinoma cell adhesion to peritoneal mesothelial cells. Am J Pathol 154 1525-1537, 1999 .

5. Strobel T and Cannistra SA: Beta1-integrins partly mediate binding of ovarian cancer cells to peritoneal mesothelium in vitro. Gynecol Oncol 73: 362-367, 1999.

6. Sawada K, Ohyagi-Hara C, Kimura T and Morishige K: Integrin inhibitors as a therapeutic agent for ovarian cancer. J Oncol 2012: 915140, 2012.

7. Hodkinson PS, Elliott T, Wong WS, Rintoul RC, Mackinnon AC, Haslett $C$ and Sethi T: ECM overrides DNA damage-induced cell cycle arrest and apoptosis in small-cell lung cancer cells through beta1 integrin-dependent activation of PI3-kinase. Cell Death Differ 13: 1776-1788, 2006.

8. Sawada K, Mitra AK, Radjabi AR, Bhaskar V, Kistner EO, Tretiakova M, Jagadeeswaran S, Montag A, Becker A, Kenny HA, et al: Loss of E-cadherin promotes ovarian cancer metastasis via alpha 5-integrin, which is a therapeutic target. Cancer Res 68: 2329-2339, 2008 .

9. Morgan MR, Byron A, Humphries MJ and Bass MD: Giving off mixed signals--distinct functions of alpha5beta1 and alphavbeta3 integrins in regulating cell behaviour. IUBMB Life 61: 731-738, 2009.

10. Marelli UK, Rechenmacher F, Sobahi TR, Mas-Moruno C and Kessler H: Tumor targeting via integrin ligands. Front Oncol 3: 222, 2013.

11. Wintzell M, Hjerpe E, Åvall Lundqvist E and Shoshan M: Protein markers of cancer-associated fibroblasts and tumor-initiating cells reveal subpopulations in freshly isolated ovarian cancer ascites. BMC Cancer 12: 359, 2012.

12. Watanabe T, Hashimoto T, Sugino T, Soeda S, Nishiyama H, Morimura Y, Yamada H, Goodison S and Fujimori K: Production of IL1-beta by ovarian cancer cells induces mesothelial cell beta1-integrin expression facilitating peritoneal dissemination. J Ovarian Res 5: 7, 2012.

13. Sawada K, Radjabi AR, Shinomiya N, Kistner E, Kenny H, Becker AR, Turkyilmaz MA, Salgia R, Yamada SD, Vande Woude GF, et al: c-Met overexpression is a prognostic factor in ovarian cancer and an effective target for inhibition of peritoneal dissemination and invasion. Cancer Res 67: 1670-1679, 2007.
14. Sun C, Li N, Yang Z, Zhou B, He Y, Weng D, Fang Y, Wu P, Chen P, Yang X, et al: miR-9 regulation of BRCA1 and ovarian cancer sensitivity to cisplatin and PARP inhibition. J Natl Cancer Inst 105: 1750-1758, 2013

15. Li M, Li J, Ding X, He M and Cheng SY: microRNA and cancer. AAPS J 12: 309-317, 2010.

16. Yi L, Zongyuan Y, Cheng G, Lingyun Z, Guilian Y and Wei G: Quercetin enhances apoptotic effect of tumor necrosis factorrelated apoptosis-inducing ligand (TRAIL) in ovarian cancer cells through reactive oxygen species (ROS) mediated CCAAT enhancer-binding protein homologous protein (CHOP)-death receptor 5 pathway. Cancer Sci 105: 520-527, 2014.

17. Lengyel E: Ovarian cancer development and metastasis. Am J Pathol 177: 1053-1064, 2010.

18. Guo W and Giancotti FG: Integrin signalling during tumour progression. Nat Rev Mol Cell Biol 5: 816-826, 2004.

19. Persad S and Dedhar S: The role of integrin-linked kinase (ILK) in cancer progression. Cancer Metastasis Rev 22: 375-384, 2003.

20. Hannigan G, Troussard AA and Dedhar S: Integrin-linked kinase: A cancer therapeutic target unique among its ILK. Nat Rev Cancer 5: 51-63, 2005.

21. Peng JM, Chen YH, Hung SW, Chiu CF, Ho MY, Lee YJ, Lai TC, Hsiao M, Liang CM and Liang SM: Recombinant viral protein promotes apoptosis and suppresses invasion of ovarian adenocarcinoma cells by targeting $\alpha 5 \beta 1$ integrin to down-regulate Akt and MMP-2. Br J Pharmacol 165: 479-493, 2012.

22. Jin $\mathrm{H}$ and Varner J: Integrins: Roles in cancer development and as treatment targets. Br J Cancer 90: 561-565, 2004.

23. Iwanicki MP, Davidowitz RA, Ng MR, Besser A, Muranen T, Merritt M, Danuser G, Ince TA and Brugge JS: Ovarian cancer spheroids use myosin-generated force to clear the mesothelium. Cancer Discov 1: 144-157, 2011.

24. Fine RN: Growth after renal transplantation in children. J Pediatr 110: 414-416, 1987.

25. Ebi H, Sato T, Sugito N, Hosono Y, Yatabe Y, Matsuyama Y, Yamaguchi T, Osada H, Suzuki $M$ and Takahashi T: Counterbalance between $R B$ inactivation and $m i R-17-92$ overexpression in reactive oxygen species and DNA damage induction in lung cancers. Oncogene 28: 3371-3379, 2009.

26. Wu Q, Luo G, Yang Z, Zhu F, An Y, Shi Y and Fan D: miR-17-5p promotes proliferation by targeting SOCS6 in gastric cancer cells. FEBS Lett 588: 2055-2062, 2014.

27. Wei Q, Li YX, Liu M, Li X and Tang H: MiR-17-5p targets TP53INP1 and regulates cell proliferation and apoptosis of cervical cancer cells. IUBMB Life 64: 697-704, 2012.

28. Gao Y, Luo LH, Li S and Yang C: miR-17 inhibitor suppressed osteosarcoma tumor growth and metastasis via increasing PTEN expression. Biochem Biophys Res Commun 444: 230-234, 2014

29. Ma Y, Zhang P, Wang F, Zhang H, Yang Y, Shi C, Xia Y, Peng J, Liu W, Yang Z, et al: Elevated oncofoetal miR-17-5p expression regulates colorectal cancer progression by repressing its target gene P130. Nat Commun 3: 1291, 2012.

30. Kesanakurti D, Chetty C, Dinh DH, Gujrati M and Rao JS: Role of MMP-2 in the regulation of IL-6/Stat3 survival signaling via interaction with $\alpha 5 \beta 1$ integrin in glioma. Oncogene 32: 327-340, 2013.

31. Bell-McGuinn KM, Matthews CM, Ho SN, Barve M, Gilbert L, Penson RT, Lengyel E, Palaparthy R, Gilder K, Vassos A, et al: A phase II, single-arm study of the anti- $\alpha 5 \beta 1$ integrin antibody volociximab as monotherapy in patients with platinum-resistant advanced epithelial ovarian or primary peritoneal cancer. Gynecol Oncol 121: 273-279, 2011. 